\title{
Methylene-blue aided rapid confocal laser endomicroscopy of breast cancer
}

\author{
Khushi Vyas \\ Michael Hughes \\ Daniel Richard Leff \\ Guang-Zhong Yang
}




\section{Methylene-blue aided rapid confocal laser endomicroscopy of breast cancer}

\author{
Khushi Vyas, ${ }^{a, *}$ Michael Hughes, ${ }^{a}$ \\ Daniel Richard Leff, ${ }^{a, b}$ and Guang-Zhong Yang ${ }^{a}$ \\ amperial College London, Hamlyn Centre for Robotic Surgery, \\ London, United Kingdom \\ ${ }^{b}$ Imperial College Healthcare NHS Trust, Imperial Breast Unit, \\ Charing Cross Hospital, London, United Kingdom
}

\begin{abstract}
Breast conserving surgery allows complete tumor resection while maintaining acceptable cosmesis for patients. Safe and rapid intraoperative margin assessment during the procedure is important to establish the completeness of tumor excision and minimizes the need for reoperation. Confocal laser endomicroscopy has demonstrated promise for real-time intraoperative margin assessment using acriflavine staining, but it is not approved for routine in-human use. We describe a custom high-speed line-scan confocal laser endomicroscopy (LS-CLE) system at $660 \mathrm{~nm}$ that enables high-resolution histomorphological imaging of breast tissue stained with methylene-blue, an alternative fluorescent stain for localizing sentinel nodes during breast surgery. Preliminary imaging results on freshly excised human breast tissue specimens are presented, demonstrating the potential of methylene-blue aided rapid LS-CLE to determine the oncological status of surgical margins in-vivo. () The Authors. Published by SPIE under a Creative Commons Attribution 3.0 Unported License. Distribution or reproduction of this work in whole or in part requires full attribution of the original publication, including its DOI. [DOI: 10.1117/1.JBO.22.2.020501]
\end{abstract}

Keywords: endomicroscopy; fluorescence; microscopy; fiber bundle; breast; surgical pathology; optical biopsy.

Paper 160733LR received Oct. 25, 2016; accepted for publication Jan. 23, 2017; published online Feb. 9, 2017.

Breast cancer is a major public health problem worldwide and the second leading cause of cancer-related female deaths. In 2012, there were an estimated 464,000 new cases of female breast cancer and 131,000 deaths in Europe alone. ${ }^{1}$ Breast conserving surgery (BCS), in the form of wide local excision with glandular remodeling, is an attractive option for patients as it allows complete tumor resection while maintaining acceptable cosmesis and the oncological outcome is similar to a mastectomy. ${ }^{2,3}$ However, on average, $\sim 20 \%$ to $30 \%$ of BCS patients require one or more reoperative interventions, mainly due to the presence of positive or close margins in postoperative histological analysis of the resected tissue.,

*Address all correspondence to: Khushi Vyas, E-mail: k.vyas13@imperial.ac .uk
For breast surgical procedures, conventional excision biopsy followed by histology imaging is still required for comprehensive assessment of margin status. While traditional histology provides high diagnostic accuracy, it is labor-intensive and slow, adding 20 to 30 min to the duration of the operation. ${ }^{6}$ One potential avenue for reducing reoperation rates following $\mathrm{BCS}$ is the use of high-resolution intraoperative imaging to determine if the radial margins are tumor-free during surgery. Optical imaging techniques, including optical coherence tomography, ${ }^{7}$ confocal reflectance microscopy,${ }^{8}$ and nonlinear microscopy, ${ }^{9}$ are currently being investigated for in-vivo visualization of breast morphology.

Probe-based confocal laser endomicroscopy (pCLE) is one such emerging optical biopsy technique that is suitable for intraoperative margin assessment as it can provide real-time imaging of tissue structure with microscopic resolution. The method typically uses a flexible optical fiber bundle to relay light to and from the tissue, and cellular features are made visible by exogenous staining prior to imaging. A preliminary study on 71 ex-vivo breast tissue samples imaged with the Cellvizio pCLE system (Mauna Kea Technologies) demonstrated that cancerous and normal tissue could be differentiated by histopathologists with an accuracy of $94 \% .^{10}$ This study used topically applied acriflavine, excited at $488 \mathrm{~nm}$, which stains cell nuclei and enables high contrast imaging of tissue morphology. However, this is not currently an attractive option for in-vivo imaging due to the lack of regulatory approval and suggested risks of mutagenicity. ${ }^{11}$

To avoid these safety concerns, a subsequent in-vivo human study used intravenous fluorescein as the contrast agent, a compound that is well-established as being safe for in-vivo human use $^{12}$ and is commonly employed for other clinical applications of pCLE ${ }^{13,14}$ However, since fluorescein staining is nonspecific, it does not highlight tissue morphology as effectively, causing difficulties in distinguishing between benign and malignant lesions. $^{12}$

For intraoperative margin assessment in routine clinical practice, it is desirable to use endomicroscopes with approved fluorescent dyes, which are both safe for in-vivo human use and provide sufficient contrast to discriminate between different tissue morphologies. In this letter, we demonstrate that normal and cancerous breast tissue stained with methylene-blue, and excited at $660 \mathrm{~nm}$, exhibits comparable morphological features to acriflavine-stained tissue. Crucially, methylene blue is a US Food and Drug Administration approved dye for the treatment of methemoglobinemia and is an alternative to isosulphan blue for in-vivo localization of nonpalpable lesions and sentinel lymph node mapping during breast surgeries. ${ }^{15-17}$ Topical methyelene blue has also been demonstrated to image precancerous and neoplastic lesions of central airways using a CLE system. ${ }^{18,19}$

Images were acquired ex-vivo using a custom high-speed line-scan confocal laser endomicroscopy (LS-CLE) system operating at $660 \mathrm{~nm}$. The system configuration is illustrated in Fig. 1(a). The system set-up is similar to a previously developed virtual slit LS-CLE at $488 \mathrm{~nm}$ but is adapted and optimized for methylene-blue imaging. A full description of the system's operating principles can be found in Ref. 20. In brief, a cylindrical lens $(f=50 \mathrm{~mm})$ is used to create a focused line from a $100 \mathrm{~mW}, 660 \mathrm{~nm}$ laser (Vortran Stradus, 660). A galvomirror (Thorlabs GVS001) sweeps the line across the proximal end of the fiber bundle probe (Cellvizio Gastroflex UHD, Mauna Kea Technologies) in a direction perpendicular to the line. 


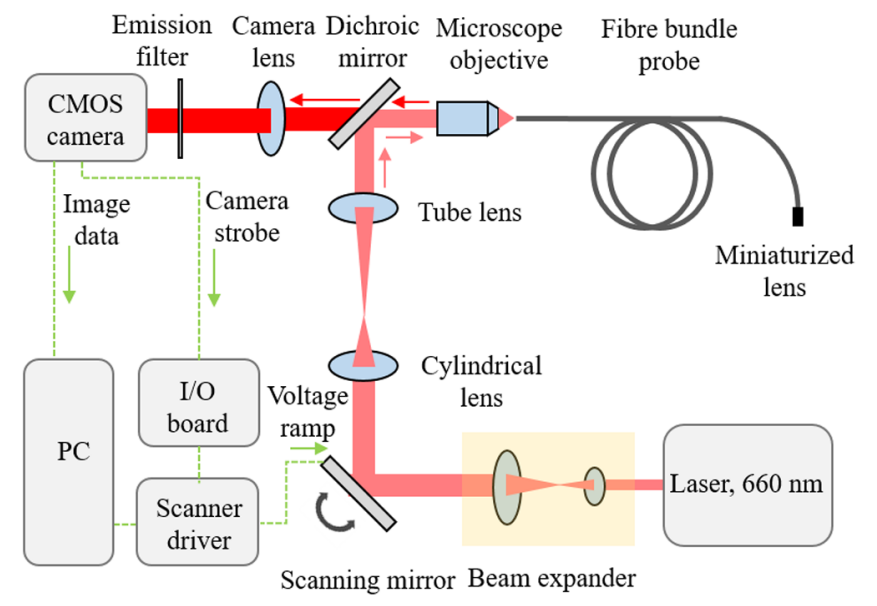

(a)

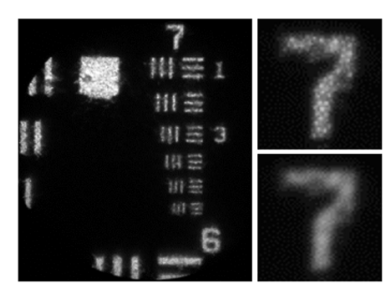

(b)

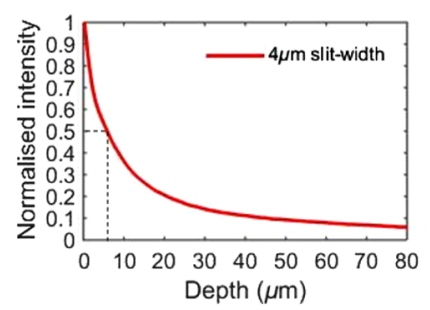

(c)
Fig. 1 (a) Schematic layout of high-speed LS-CLE system operating at $660 \mathrm{~nm}$. (b) Image of high-resolution USAF resolution target showing group 7 elements 1-6 when back-illuminated with a red LED. (c) Axial sectioning profile for the line-scanning system.

The fiber bundle probe relays the line to the tissue via a distal objective and returns collected fluorescence from all the points along the line, which is then imaged onto a monochrome rollingshutter CMOS camera (Flea 3, FL3-U3-13S2M-CS). In this design, the rolling shutter of the CMOS camera operates as a virtual detector slit that rejects most of the out-of-focus light, leading to optical sectioning at frame rates of up to $120 \mathrm{~Hz}$. To accommodate the $660-\mathrm{nm}$ excitation wavelength, the dichroic and emission filters from the system reported previously ${ }^{20}$ were replaced with a Thorlabs-DMLP650R longpass dichroic mirror and a Semrock BLP01-664R-25 longpass edge filter, respectively.

The spatial resolution of the system was measured by backilluminating a 1951 USAF resolution target with a red LED; Fig. 1(b) shows an image of group 7 of the target. For realtime visualization, pixelation artifacts due to the fiber cores were removed by convolution with a 2-D Gaussian filter ( $\sigma=1.6$ pixels, $1.4 \mu \mathrm{m}$ on the proximal face of the bundle). Figure 1(b) shows an image of the numeral "7" of the resolution target before (top) and after (bottom) removal of pixelation artifacts. Figure 1(c) demonstrates the axial sectioning strength of LS-CLE for detector slit-width of $4 \mu \mathrm{m}$ (measured as described in Ref. 20). For this study, a slit width of $4 \mu \mathrm{m}$ was used to give an axial resolution of $\sim 11 \mu \mathrm{m}$. While point-scanning pCLE would probably be needed for intravenous fluorescein imaging (see Ref. 20 for a discussion), our partially sectioning system is sufficient for imaging tissue sites topically stained with methylene blue and provides the advantage of a much higher frame rate. The high frame rate enables image mosaics to be assembled more readily, ${ }^{21}$ which, as discussed in Ref. 10 , is important for allowing features to be identified with confidence.
A total of 15 freshly excised tissue samples were acquired from five patients, who underwent breast surgery between March and August, 2016, under an Imperial College tissue bank license (Project R12047). Several small cut-outs, approximately $10 \times 10 \mathrm{~mm}$ in size, were taken from normal and diseased sections (as subsequently confirmed by histology) and imaged using the following protocol $\sim 30$ to $45 \mathrm{~min}$ after excision.

Methylene blue could be applied topically for in vivo imaging, as demonstrated in Ref. 19. For this ex-vivo study, methylene blue solution $(0.1 \%$ in saline; Sigma Aldrich, United Kingdom) was applied topically to each cut-out for $30 \mathrm{~s}$ followed by a gentle wash with PBS saline to remove excess stain. The tip of the endomicroscope probe was gently applied to the tissue surface. At the end of the imaging procedure, excess methylene-blue dye was gently wiped off the surface of the tissue, and the specimen was returned to histology for routine analysis.

In postprocessing, a mosaicking algorithm was used to stitch overlapping frames, producing a larger image more comparable to histology slides. This algorithm, described fully in Ref. 21 and based on an established procedure, ${ }^{22}$ uses two-way fast normalized cross-correlation (NCC) for pairwise image registration and combines images using distance-weighted alpha-blending. Mosaics were then stored digitally in a prospectively maintained database. To assist with probe scanning during image acquisition, a real-time preview of the mosaics, using one-way NCC and dead-leaf blending, was displayed to the operator.

The accuracy of normal/tumor identification using acriflavine staining pCLE has already been established $;^{10}$ so, the main purpose of this pilot study was to determine whether the 660-nm system could identify features following the taxonomy of pathologies developed in the previous study. A total of 450 pCLE image mosaics (mean 30 per sample; range 20 to 40 ) were generated from 15 specimens. Since we have a limited number of tissue samples, we acquired many more mosaics than necessary for detailed performance assessment. Figure 2 provides representative LS-CLE image mosaics of freshly excised normal, benign, and neoplastic human breast specimens stained with methylene blue. The potential of the approach to identify the microscopic tissue morphology relevant to tumor identification is demonstrated by comparison of the reconstructed mosaics with those from the previously generated data bank generated using the Cellvizio pCLE with acriflavine $(0.02 \%)$ staining, and with conventional histology. ${ }^{10}$

Two features typifying normal breast tissue were identified: adipose tissue and the presence of collagen fibres in the stroma. As shown in Figs. 2(a) and 2(b), the fluorescence images obtained from methylene-blue and acriflavine-stained adipose tissue are similar: the well-defined and uniform nonfluorescent polygon shape of the adipose tissue with hyperfluorescent borders can clearly be observed in both cases. Nuclei, which may not always be clearly visible, appear as sparsely populated bright spots on the borders. In Figs. 2(d) and 2(e), the fibrous connective tissue appears as hyperfluorescent bundles of elastic wavy fibres. Figures 2(g) and 2(h) represent fibrocystic changes, where compressed bright slit-like glandular spaces are surrounded by loose fibrous stroma. In Figs. 2(j) and 2(k), stroma composed of hyperfluoroscent spindle-shaped fibroblasts appear surrounded by gray collagen fibers. Also, both the systems enable visualization of distinctive neoplastic changes characterized by increased cellularity in the stroma, as depicted in Figs. 2(m) and 2(n). 


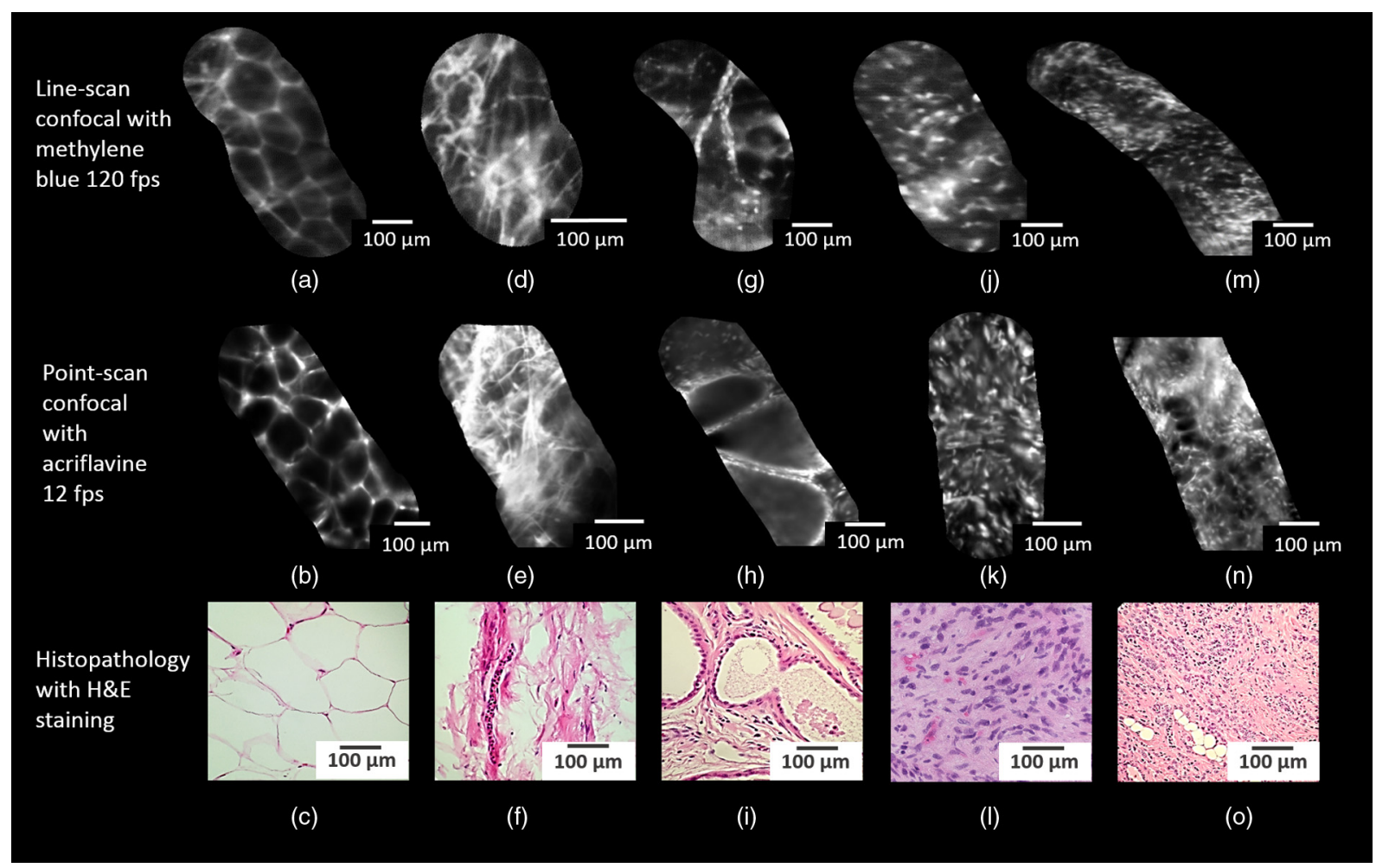

Fig. 2 (a, d, g, j, and $m$ ) Non-neoplastic and neoplastic breast tissue imaged with LS-CLE with methylene-blue, (b, e, h, k, and n) point-scan pCLE with acriflavine, and (c, f, i, l, and o) conventional histology with H\&E staining. Non-neoplastic features shown are adipose tissue (first column) and fibrous connective tissue (second column). Benign fibrocystic changes are shown in third column and spindle-shaped fibroblasts are shown in the fourth column. Invasive cancer of the breast is shown in the fifth column. Scale bar corresponds to $100 \mu \mathrm{m}$. H\&E images have been size matched to the $100 \mu \mathrm{m}$ scale bar.

The disorganized distribution of cell nuclei and the unclear tissue architecture was clearly visible as large clusters of hyperfluorescent spots. Similar features are observed in matched histology images of acriflavine-stained tissue (c, f, i, l, and o). Furthermore, we compared the signal-to-noise ratio (SNR) of the two sets of images, obtained by the ratio of the mean intensity of a homogenous stained area of $10 \times 10$ pixels of the tissue (signal) to the standard deviation of an unstained region of $10 \times 10$ pixels (noise). Averaged across 100 images, the SNR was $(179 \pm 10)$ for the acriflavine-stained images and $(170 \pm 13)$ for the methylene-blue stained images. Therefore, despite the differences in stain, imaging system and processing, the noise in the final processed images are broadly comparable.

In summary, a high-speed 660-nm LS-CLE system has been used with topical application of $0.1 \%$ methylene blue to demonstrate rapid morphological assessment of ex-vivo breast cancer tissues. Images and mosaics of normal and neoplastic breast tissue, acquired with a lateral resolution of $2.2 \mu \mathrm{m}$ at $120 \mathrm{~Hz}$, showed distinctive morphological features. The methylene blue aided LS-CLE produced images with comparable contrast to point-scanning pCLE with acriflavine staining but with the advantage of a higher frame rate and use of a safe and regulatory-approved (off-label) stain.

These current results suggest a clinical application for methylene-blue aided LS-CLE in breast cavity scanning for rapid decision-making during $\mathrm{BCS}$, allowing oncological status of resection margins to be determined intraoperatively. This could allow the surgeon to accomplish more accurate and complete surgical resections, thus reducing the reoperation rates and improving outcomes. Further work will be required to confirm that the quantitative accuracy when using methylene-blue staining is similar to that when using acriflavine and to establish whether similar images can be obtained in-vivo.

\section{Disclosures}

Ms. Vyas, Dr. Hughes, Mr. Leff, and Prof. Yang have nothing to disclose. No conflicts of interest, financial or otherwise, are declared by the authors.

\section{Acknowledgments}

We would like to thank Petros Giataganas, Dr. Tou-Pin Chang, Professor Sami Shousha and Dr. Rathi Ramakrishnan for useful discussions and Imperial College Tissue bank team for providing the tissue samples. This work was supported by EPSRC Grant EP/I027769/1: SMART Endomicroscopy and EP/ N022521/1: SMART Endomicroscopy Translational Alliance.

\section{References}

1. J. Ferlay et al., "Cancer incidence and mortality patterns in europe: estimates for 40 coun-tries in 2012," Eur. J. Cancer 49(6), 1374-1403 (2013).

2. M. S. Moran et al., "Society of surgical oncology-American society for radiation oncology consensus guideline on margins for breast-conserving surgery with whole-breast irradiation in stages I and II invasive breast cancer," Int. J. Radiat. Oncol. Biol. Phys. 88(3), 553-564 (2014).

3. S. Krämer et al., "Breast-conserving treatment of breast canceroncological and reconstructive aspects," Gynäkologisch-geburtshilfliche Rundschau 48(2), 56-62 (2008). 


\section{JBO Letters}

4. R. Jeevan et al., "Reoperation rates after breast conserving surgery for breast cancer among women in England: retrospective study of hospital episode statistics," Br. Med. J. 345, e4505, (2012).

5. R. G. Pleijhuis et al., "Obtaining adequate surgical margins in breastconserving therapy for patients with early-stage breast cancer: current modalities and future directions," Ann. Surg. Oncol. 16(10), 2717-2730 (2009).

6. S. J. Erickson-Bhatt et al., "Real-time imaging of the resection bed using a handheld probe to reduce incidence of microscopic positive margins in cancer surgery," Cancer Res. 75(18), 3706-3712, (2015).

7. E. F. Brachtel et al., "Spectrally encoded confocal microscopy for diagnosing breast cancer in excision and margin specimens," Lab. Invest. 96(4), 459-467 (2016).

8. Y. K. Tao et al., "Assessment of breast pathologies using nonlinear microscopy," Proc. Nat. Acad. Sci. 111(43), 15304-15309, (2014).

9. E. R. St John et al., "Diagnostic accuracy of intraoperative techniques for margin assessment in breast cancer surgery: a meta-analysis," Ann. Surg. 265(2), 300-310 (2017).

10. T. P. Chang et al., "Imaging breast cancer morphology using probebased confocal laser endomicroscopy: towards a real-time intraoperative imaging tool for cavity scanning," Breast Cancer Res. Treat. 153(2), 299-310 (2015).

11. R. Kiesslich et al., Atlas of Endomicroscopy, Springer Science \& Business Media, Berlin, Germany (2007).

12. G. D. De Palma et al., "Confocal laser endomicroscopy in breast surgery: a pilot study," BMC Cancer 15(1), 1 (2015).

13. F. S. Fuchs et al., "Fluorescein-aided confocal laser endomicroscopy of the lung," Respiration 81(1), 32-38 (2011).
14. S. Paramsothy et al., "Endoscopy: fluorescein contrast in confocal laser endomicroscopy," Nat. Rev. Gastroenterol. Hepatol. 7(7), 366-368 (2010).

15. J. M. East et al., "Sentinel lymph node biopsy for breast cancer using methylene blue dye manifests a short learning curve among experienced surgeons: a prospective tabular cumulative sum (cusum) analysis," $B M C$ Surg. 9(1), 1 (2009).

16. A. Nasrinossadat et al., "Marking non-palpable breast masses with injected methylene blue dye, an easy, safe and low cost method for developing countries and resource-limited areas," Asian Pac. J. Cancer Prev. 12, 1189-1192 (2011).

17. J. Tang et al., "Significance of methylene blue dye for localization biopsy of nonpalpable breast lesions," Chin. J. Cancer 28, 775-782 (2009).

18. L. Thiberville et al., "Confocal fluorescence endomicroscopy of the human airways," Proc. Am. Thorac. Soc. 6(5), 444-449 (2009).

19. T. Lai et al., "Safety and performance analysis of acriflavine and methylene blue for in vivo imaging of precancerous lesions using fibered confocal fluorescence microscopy (FCFM): an experimental study," $B M C$ Pulm. Med. 15(1), 1 (2015).

20. M. Hughes et al., "Line-scanning fiber bundle endomicroscopy with a virtual detector slit," Biomed. Opt. Exp. 7(6), 2257-2268 (2016).

21. M. Hughes et al., "High speed, line-scanning, fiber bundle fluorescence confocal endomicroscopy for improved mosaicking," Biomed. Opt. Exp. 6(4), 1241-1252 (2015).

22. T. Vercauteren et al., "Real time autonomous video image registration for endomicroscopy: fighting the compromises," Proc. SPIE 6861, 68610C (2008). 